\title{
Sox5 contributes to prostate cancer metastasis and is a master regulator of TGF- $\beta$-induced epithelial mesenchymal transition through controlling Twist1 expression
}

Jieping $\mathrm{Hu}^{1,2,4}$, Jing Tian ${ }^{1,4}$, Shimiao Zhu ${ }^{1,4}$, Libin Sun ${ }^{1,3}$, Jianpeng $\mathrm{Yu}^{1}$, Hao Tian ${ }^{1}$, Qian Dong ${ }^{1}$, Qiang Luo ${ }^{1}$, Ning Jiang ${ }^{1}$, Yuanjie Niu ${ }^{*, 1}$ and Zhiqun Shang ${ }^{\star, 1}$

${ }^{1}$ Tianjin Institute of Urology, the Second Hospital of Tianjin Medical University, Pingjiang Rd 23\#, Hexi District, Tianjin 300211, China; ${ }^{2}$ Department of Urology, the First Affiliated Hospital of Nanchang University, Jiangxi 330000, China and ${ }^{3}$ Department of Urology, First Affiliated Hospital, Shanxi Medical University, Shanxi 030001, China

Background: Metastatic castration-resistant prostate cancer (mCRPC) is one of the main contributors to the death of prostate cancer patients. To date, the detailed molecular mechanisms underlying mCRPC are unclear. Given the crucial role of epithelialmesenchymal transition (EMT) in cancer metastasis, we aimed to analyse the expression and function of Transforming growth factor-beta (TGF- $\beta$ ) signal-associated protein named Sox5 in mCRPC.

Methods: The protein expression levels were analysed by western blot, immunohistochemistry and immunofluorescence. Luciferase reporter assays and chromatin immunoprecipitation were employed to validate the target of Sox5. The effect of Smad3/Sox5/Twist1 on PCa progression was investigated in vitro and in vivo.

Results: Here, we found that TGF- $\beta$-induced EMT was accompanied by increased Sox 5 expression. Interestingly, knockdown of Sox 5 expression attenuated EMT induced by TGF- $\beta$ signalling. Furthermore, we demonstrated that Smad 3 could bind to the promoter of Sox 5 and regulate its expression. Mechanistically, Sox 5 could bind to Twist1 promoter and active Twist1, which initiated EMT. Importantly, knockdown of Sox5 in prostate cancer cells resulted in less of the mesenchymal phenotype and cell migration ability. Furthermore, targeting Sox 5 could inhibit prostate cancer progression in a xenograft mouse model. In clinic, patients with high Sox5 expression were more likely to suffer from metastases, and high Sox5 expression also has a lower progression-free survival and cancer specific-survival in clinic database.

Conclusions: Therefore, we propose a new mechanism in which Smad3/Sox5/Twist1 promotes EMT and contributes to PCa progression.

\footnotetext{
*Correspondence: Professor Z Shang; E-mail: zhiqun_shang@tmu.edu.cn or Professor Y Niu; E-mail: niuyuanjie9317@163.com

${ }^{4}$ These authors contributed equally to this work.
}

Received 6 July 2017; revised 12 September 2017; accepted 26 September 2017; published online 9 November 2017

(C) 2018 Cancer Research UK. All rights reserved 0007-0920/18 
It is estimated that 161360 new cases of prostate cancer (PCa) were diagnosed and that 26730 people died of PCa in 2017 (Siegel et al, 2017). Five-year survival rates can be $100 \%$ when PCa is localised; however, once it has spread, the chances of survival are only $28 \%$ (Hodson, 2015), and $34.4 \%$ of patients were presented with metastatic disease at diagnosis (Steinberger et al, 2016). Metastases are present in at least $80 \%$ of castration-resistant prostate cancer (CRPC) patients (Hussain et al, 2016). Cancer metastasis is a multistep process. First, metastases initiate as cancer cells and acquire invasive potential; cancer cells then grow expansively and invade the basement membrane in surrounding tissues, accompanied by angiogenesis; cancer cells eventually become circulating tumour cells (CTCs) and are transported around body; finally, CTC arrest and extravasations to secondary tissues or organs occur to form micro- or macro-metastases (Jiang et al, 2015). Epithelial-mesenchymal transition (EMT) has been shown to play a critical role in the acquisition of invasive potential, thereby promoting metastasis (Thiery and Lim, 2013).

EMT signifies the conversion of polarised immobile epithelial cells into spindle-shaped motile mesenchymal cells and enables cell migratory capacity and invasiveness (Kalluri and Weinberg, 2009; Tsai and Yang, 2013; Jadan et al, 2015). The transforming growth factor- $\beta$ (TGF- $\beta$ ) signalling pathway is a potent inducer of EMT and plays a key role in cancer metastases (Massague, 2008). SMADmediated canonical signalling and SMAD-independent noncanonical signalling are then required for its downstream signalling activation. Smad2/3 phosphorylation and nuclear translocation are involved in canonical signalling, and the nuclear-localised SMAD complex initiates transcriptional activation or transcriptional repression of several genes (Pickup et al, 2013). Transforming growth factor-beta inhibition has shown certain clinical effects in lung cancer and hepatic cell carcinoma, suggesting that inhibition of TGF- $\beta$ signalling is an emerging strategy for cancer therapy (Neuzillet et al, 2015). However, the mechanisms by which TGF- $\beta$ promotes cancer metastasis are largely unknown.

SRY-related high-mobility-group box 5 (Sox5) is a member of the Sox family, consisting of more than 20 Sox genes falling into groups $\mathrm{A}$ to $\mathrm{H}$, and belongs to the Sox D group (Lefebvre et al, 2007). Previous studies of Sox 5 function focus mainly on chondrogenic differentiation (Lefebvre et al, 2001; Ikeda et al, 2005). Recently, research shows that Sox 5 enhances progression of various tumours such as nasopharyngeal carcinoma, pituitary tumour, hepatocellular carcinoma, and breast cancer (Huang et al, 2008; Pei et al, 2014; Renjie W and Haiqian L, 2015; Wang et al, 2015). Its functions in PCa have not been explored yet. Here, we report that Sox 5 is responsible for TGF- $\beta$-induced EMT and that Smad3-Sox5-Twist1 signalling acts as an axis to promote EMT and contribute to prostate cancer metastasis.

\section{MATERIALS AND METHODS}

Cell culture and transfection. CW22RV1, PC-3 and LNCaP cell lines were maintained in RPMI 1640 media containing penicillin ( 25 units $\mathrm{ml}^{-1}$ ), streptomycin $(25 \mathrm{~g} \mathrm{ml}$ ), and $10 \%$ foetal bovine serum (FBS). Cos-1 cell line was maintained in Dulbecco's modified Eagle's medium (Invitrogen, Shanghai, China) containing 10\% FBS. Cells were incubated at $37^{\circ} \mathrm{C}$ with $5 \% \mathrm{CO}_{2}$. For knocking down Sox 5 (Gene Copoeia, Guangdong Province, PR China, Catalogue\#HSH017632-mH1), X-tremeGENE HP DNA Transfection Reagent (Roche, Shanghai, China) was used. We seeded cells in sixwell plates and grew them to $70-90 \%$ confluence for transfection. We prepared plasmid DNA-Transfection Reagent complexes (each well required $2000 \mathrm{ng}$ DNA and $6 \mu \mathrm{l}$ Transfection Reagent), incubated the complexes at room temperature for $25 \mathrm{~min}$, and then added to cells. After $48 \mathrm{~h}$ transfection, cells were collected for experiments. Western blot assays were used to confirm the infection efficiency.
Immunohistochemistry. Tumour tissues were fixed in $4 \%$ neutral buffered paraformaldehyde and embedded in paraffin. The primary antibodies of the rabbit anti-Sox5 (Bioss Biosynth, Beijing, China, bs-17136R), the mouse anti-N-cadherin (Abcam, Shanghai, China, Catalogue\# 124397), and the mouse anti-E-cadherin (BD biosciences, Beijing, China, Catalogue\# 610181) were used for staining. The primary antibody was recognised by secondary antibody (ZSGB-BIO, PV-6000), and visualised by DAB kit (ZSGB-BIO, ZLI-9019). The IHC scores were calculated as reported with modification to normalise the score (Xie et al, 2015).

RNA isolation and quantitative analysis. Total cellular RNAs were extracted by TRIzol reagent (Invitrogen) according to the manufacturer's instructions. Total RNA (1 ng) was used to synthesise first strand complementary DNA by Revert Aid First Strand cDNA Synthesis Kit (Thermo scientific, Shanghai, China, \#K 1622) according to the manufacturer's protocol. The amount of certain cDNA was measured in real-time PCR assay using Fast Start Universal SYBR Green Master (Roche). Primers were: Sox5 Forward: 5'-AGGTTTGGACTCACTTGACAGG-3', Reverse: 5'-TCCATCTG CTTCCCCATACG-3'; Twist1 Forward: 5'-AGCTACGCCTTCT CCGTCTG-3', Reverse: 5'-CTCCTTCTCTGGAAACAATGACA-3'; and GAPDH Forward: $5^{\prime}$-TGGCTTCATAGG TGACTTCCA-3', Reverse: 5'-AAGGACCTGTCTAGGTTTGATGC-3'

Western blot analysis. Harvested cells were washed with PBS and lysed in RIPA buffer (50 mm Tris- $\mathrm{HCl} / \mathrm{pH} 7.4 ; 1 \% \mathrm{NP}-40 ; 150 \mathrm{~mm}$ $\mathrm{NaCl} ; 1 \mathrm{~mm}$ EDTA; $1 \mathrm{~mm}$ proteinase inhibitor; $1 \mathrm{~mm} \mathrm{Na} \mathrm{VO}_{4} ; 1 \mathrm{~mm}$ $\mathrm{NaF}$; $1 \mathrm{~mm}$ okadaic acid; and $1 \mathrm{mg} \mathrm{ml}^{-1}$ aprotinin, leupeptin, and pepstatin). Samples (30 $\mu$ g protein) were separated on $8 \%$ SDSPAGE gel and transferred to PVDF membranes at $4{ }^{\circ} \mathrm{C}(250 \mathrm{~mA}$, $2 \mathrm{~h}$ ). Membranes were blocked in 5\% fat-free milk in TBST for $1 \mathrm{~h}$ at room temperature, and incubated with appropriate diluted primary antibodies GAPDH (Sungene Biotech, Tianjin, China, KM9002, 1:5000), Sox5 (Bioss Biosynth, bs-17136R, 1:1000), N-cadherin (Abcam, Catalogue\# 124397, 1:1000), Twist1 (Abcam, ab50887, $1: 50$ ), Histone 3 (Abcam, ab8580, 1:1000), and E-cadherin (BD biosciences, Catalogue\# 610181, 1:1000) were used for staining overnight at $4{ }^{\circ} \mathrm{C}$, then washed $10 \mathrm{~min}$ three times, and incubated with HRP-conjugated anti-rabbit or anti-mouse antibody for $1 \mathrm{~h}$ at room temperature, washed $10 \mathrm{~min}$ three times. The blots were developed in ECL mixture and visualised by Imager.

Migration assay. Cells (105for LNCaP and CWR22RV1, $5 \times 104$ for PC-3) after different treatments were re-suspended with serumfree media and seeded in the upper chambers of the transwells. Foetal bovine serum (10\%) with or without $10 \mathrm{ng} \mathrm{ml}^{-1}$ TGF- $\beta 1$ (R\&D Systems, Shanghai, China) was put in the lower chambers. After $24 \mathrm{~h}$ (PC-3) or $48 \mathrm{~h}$ (LNCaP and CWR22RV1) incubation, the cells invaded to the lower part of the membrane were harvested, fixed with $75 \%$ ethanol, and stained with $0.1 \%$ crystal violet (Solarbio, Beijing, China, G1061) in PBS. The invaded cells were counted under microscope. The s.d. was calculated from three independent wells.

Nuclear protein extraction. We prepared cytoplasmic and nuclear extracts as described elsewhere ( $\mathrm{Wu}, 2006)$. Before the procedure, following reagents were prepared and stored in stock concentrations. $0.5 \mathrm{M}$ sodium fluoride (NaF; Sigma, Shanghai, China), stored at $4{ }^{\circ} \mathrm{C} ; 100 \mathrm{~mm}$ phenylmethylsulphonyl fluoride (PMSF; Sigma) solution in isopropanol, stored at $-20^{\circ} \mathrm{C} ; 0.1 \mathrm{M}$ dithiotreitol (DTT; Invitrogen), stored at $-20^{\circ} \mathrm{C} ; 1 \mathrm{mg} \mathrm{ml}^{-1}$ leupeptin (Sigma), stored at $-20^{\circ} \mathrm{C} ; 1.25 \mathrm{M} \beta$-glycerophosphate disodium salt (Sigma), stored at $4{ }^{\circ} \mathrm{C} ; 1 \mathrm{M}$ sodium vanadate (Sigma), stored at $-20^{\circ} \mathrm{C} ; 1 \mathrm{~m}$ potassium chloride (KCl; Aldrich, Milwaukee, WI, USA) stored at room temperature (RT); $1 \mathrm{M}$ HEPES (Sigma), stored at $4{ }^{\circ} \mathrm{C} ; 1 \mathrm{M}$ magnesium chloride hexahydrate ( $\mathrm{MgCl} 2$, Sigma), stored at RT; $2 \mathrm{M}$ sucrose (Sigma), stored at 
RT; 10\% Igepal CA-630 (NP-40; Sigma), stored at RT; 5 M sodium chloride (NaCl; Sigma), stored at RT; and $0.5 \mathrm{~m}$ EDTA (Invitrogen), stored at RT. Then the compound was prepared, buffer A: $10 \mathrm{~mm}$ HEPES, pH 7.9, $1.5 \mathrm{~mm} \mathrm{MgCl}_{2}, 10 \mathrm{~mm} \mathrm{KCl}, 300 \mathrm{~mm}$ sucrose, $0.5 \%$ NP-40, stored at $4{ }^{\circ} \mathrm{C}$; buffer B: $20 \mathrm{~mm}$ HEPES, pH 7.9, $1.5 \mathrm{mM} \mathrm{MgCl}_{2}, 420 \mathrm{~mm} \mathrm{NaCl}, 0.2 \mathrm{~mm}$ EDTA, $2.5 \%$ glycerol, stored at $4{ }^{\circ} \mathrm{C}$; and buffer D: $20 \mathrm{~mm}$ HEPES, pH 7.9, $100 \mathrm{~mm} \mathrm{KCl}, 0.2 \mathrm{~mm}$ EDTA, $8 \%$ glycerol, stored at $4{ }^{\circ} \mathrm{C}$. Medium from cultured cells were removed and washed with cold PBS, cells were harvested with a rubber scraper, centrifuged at $550 \mathrm{~g}$ for $5 \mathrm{~min}$, and then the supernatant was discarded. To buffers $\mathrm{A}, \mathrm{B}$, and $\mathrm{D}$, the following inhibitors were added: $0.5 \mathrm{~mm}$ PMSF, $1 \mathrm{~mm} \mathrm{Na}_{3} \mathrm{VO}_{4}, 0.5 \mathrm{~mm}$ DTT, $1 \mu \mathrm{g} \mathrm{ml}^{-1}$ leupeptin, $25 \mathrm{~mm} \beta$-glycerophosphate, and $10 \mathrm{~mm} \mathrm{NaF}$. the pellet in two package cell volume of buffer A with inhibitors was resuspended and kept on ice for $10 \mathrm{~min}$, vortexed briefly, and centrifuged at $2600 \mathrm{~g}$ for $30 \mathrm{~s}$. The supernatant was collected and labelled as cytoplasm protein. The pellet in $2 / 3$ package cell volume of buffer B with inhibitors was resuspended. The mixture was resonicated for $5 \mathrm{~s}$ and centrifuged at $10400 \mathrm{~g}$ for $5 \mathrm{~min}$. The supernatant with equal volume of buffer D with inhibitors was diluted and labelled nuclear protein.

Immunofluorescence. The cells were seeded on coverslips in sixwell culture plates. After various treatments, the cells were fixed (15 min) with $4 \%$ paraformaldehyde, permeabilised $(5 \mathrm{~min})$ with
PBS containing $0.1 \%$ Triton X-100, and fixed (10 min) with $4 \%$ paraformaldehyde. Then the cells were blocked with goat serum (BOSTER) for $1 \mathrm{~h}$ at $37^{\circ} \mathrm{C}$, antibodies Sox $5, \mathrm{~N}$-cadherin, or Twist 1 were added, incubated at $4{ }^{\circ} \mathrm{C}$ overnight. After incubation with affinity-purified antibody Cy5 labelled goat anti-rabbit $\operatorname{IgG}(\mathrm{H}+\mathrm{L})$ and affinity-purified antibody Dylight 488 labelled goat antimouse/rabbit IgG $(\mathrm{H}+\mathrm{L})$ (Kirkegaard\&Perry Laboratories, Shanghai, China) at $37^{\circ} \mathrm{C}$ for $1 \mathrm{~h}$, the cells were washed three times with PBS and stained with DAPI. Images were acquired using confocal microscope with Olympus fluo view 4.0 version.

Chromatin immunoprecipitation (CHIP). LNCaP cells were treated with $10 \mathrm{ng} \mathrm{ml}^{-1}$ TGF- $\beta 1$ for $24 \mathrm{~h}$ to perform Smad3 (Abcam, ab28379) CHIP assay using EpiQuik Chromatin Immunoprecipitation Kit (Epigentek, Farmingdale, NY, USA ) according to the protocol. CW22RV1 cells were used to perform Sox5 (Abcam, ab94396) CHIP assay. PCR was performed using primers specific for the Smad3 or Sox 5 binding region in the Sox 5 or Twist1 promoter. Sox5 promoter-Forward: 5'-AGTATGGGAGACGTGTTAAATGAGT-3'.

Sox 5 promoter-Reverse: 5'-ACTTCCAGCAGCGGAGTCTG-3'. Twist1 promoter-Forward: 5'-CTTAGGCGCTATCAAATTCCC3', Twist1 promoter-Reverse: 5'-AGCGACAGCAGCAATGGCAAC- $3^{\prime}$.
A

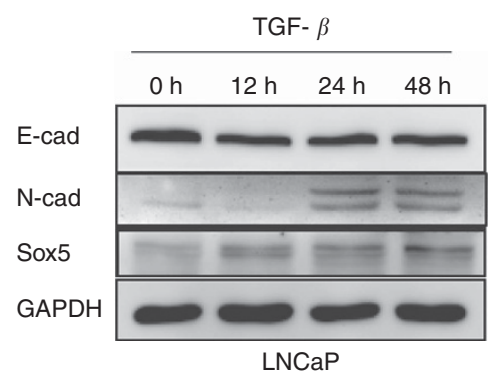

C

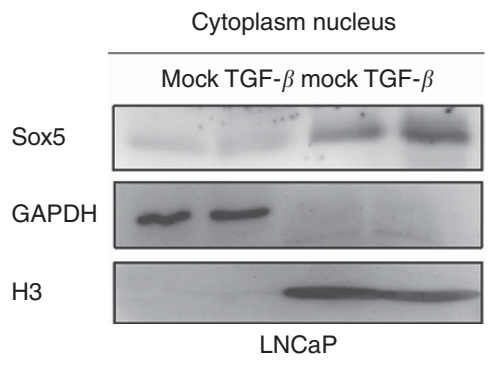

B

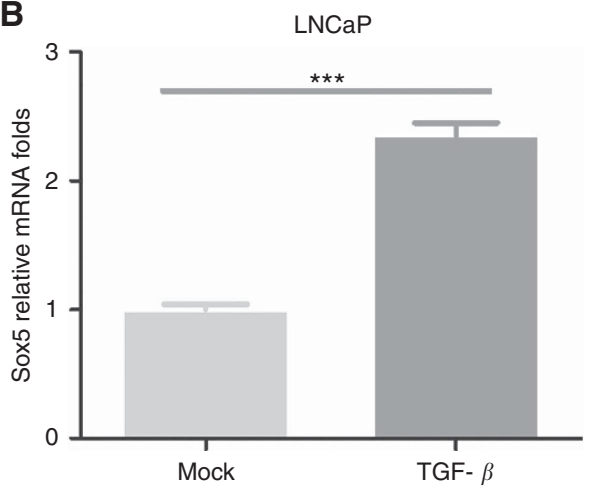

D

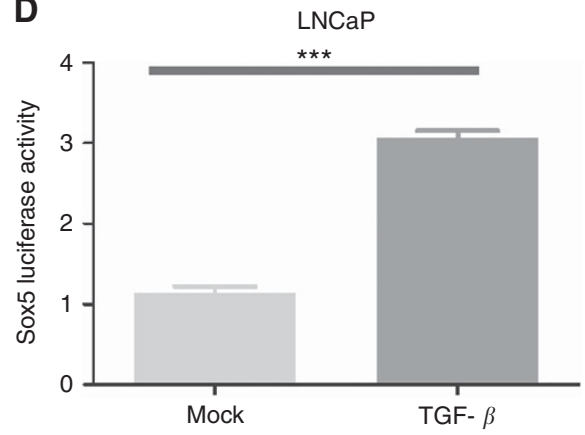

E

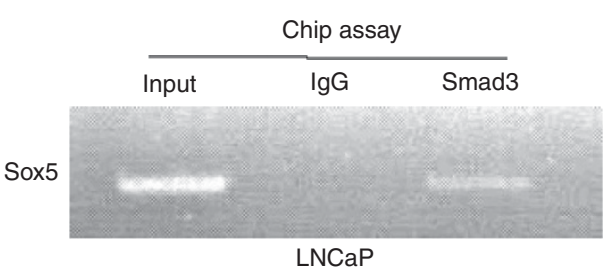

Figure 1. Sox 5 is induced by TGF- $\beta$. (A) LNCaP cells were treated with $10 \mathrm{ng} \mathrm{ml}^{-1} \mathrm{TGF}-\beta$, whole cell lysates were extracted at the indicated time, E-cadherin, N-cadherin, and Sox5 were measured by immunoblotting, and GAPDH was used as a loading control. (B) LNCaP treated with vehicle or $10 \mathrm{ng} \mathrm{ml}^{-1}$ TGF- $\beta$ for $24 \mathrm{~h}$. Sox 5 mRNA fold change was measured by Q-PCR. (C) LNCaP treated with vehicle or $10 \mathrm{ng} \mathrm{ml}^{-1} \mathrm{TGF}-\beta$ for $24 \mathrm{~h}$. Cytoplasm and nuclear protein were extracted and measured by immunoblotting. (D) Immunofluorescence showed Sox 5 expression in LNCaP treated with vehicle or TGF- $\beta$. (E) LNCaP transfected with Sox5 luciferase plasmid and treated with vehicle or TGF- $\beta$. Luciferase values were measured. ${ }^{\star \star \star} P<0.001$. 
A

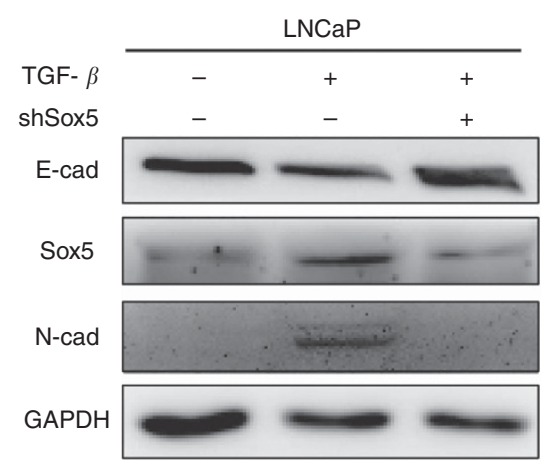

B

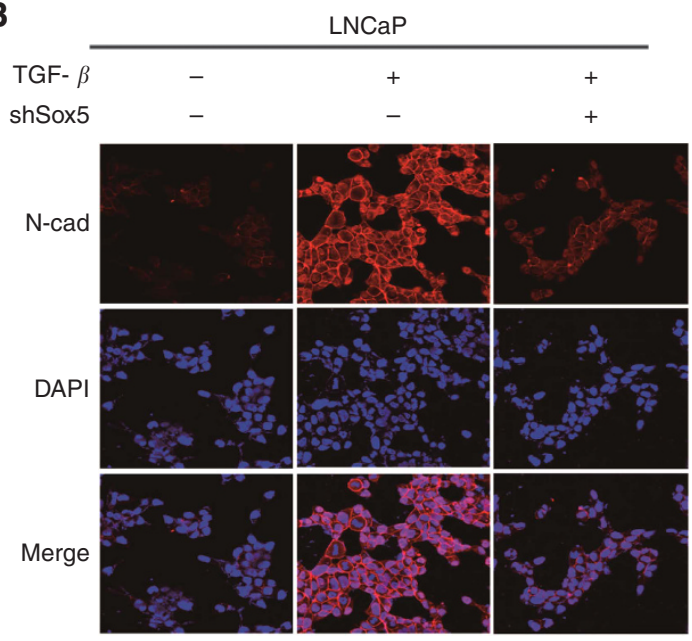

D

C

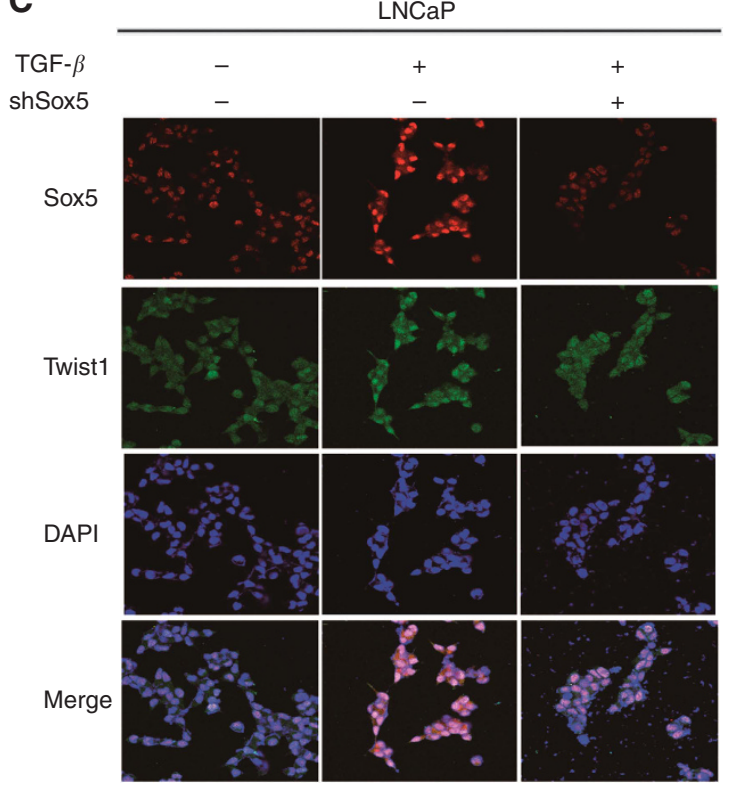

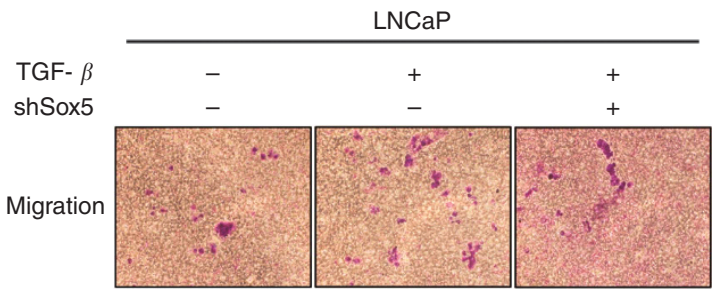

E

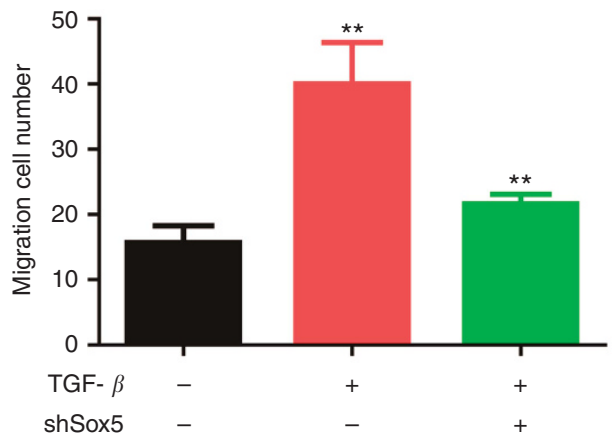

Figure 2. Sox 5 is responsible for TGF- $\beta$-induced EMT. (A) LNCaP cells were transfected with vehicle or shSox 5 plasmid, and then treated with vehicle or TGF- $\beta$. E-cadherin, $\mathrm{N}$-cadherin and Sox 5 expression were detected by immunoblotting. (B and $\mathbf{C}$ ) The expression of $\mathrm{N}$-cadherin and Twsit1 were detected by immunofluorescence. (D) The mobility of LNCaP cells with the indicated treatment was measured by migration assays (E). The migrated cells were counted. ${ }^{\star \star} P<0.01$.

Luciferase reporter assays. Primers with restriction enzyme sites HindIII/NheI were designed to amplify the promoter fragment of Sox 5 or Twist1 from genomic DNA with a length of 459 or $626 \mathrm{bp}$. DNA fragments were cloned into pGL4.27 promoter luciferase vector (Promega, Beijing, China). LNCaP cells were transfected with the Sox 5 luciferase reporter constructs; Cos- 1 cells were transfected with the Twist1 luciferase reporter constructs with or without Sox 5 over express plasmid (Gene Copoeia, Catalogue\#EX-Z4528-Lv105-5). Supernatants were collected $24 \mathrm{~h}$ after stable transfection. DualLuciferase Reporter Assay System (Promega) was applied to measure luciferase value according to the manufacturer's instructions.

Xenograft mice model. Male 6- to 8-week-old nude mice were purchased from Beijing HFK Bioscience Co. Ltd. (Beijing, China). The CWR22RV1 cells transfected with vehicle or shSox5 plasmid were injected subcutaneous with matrigel. About 3 weeks later, the tumour was detached and cut into pieces around $1 \times 1 \times 1 \mathrm{~mm}$ size. Tumours were implanted orthotopically in the anterior prostates of the mice, and also subcutaneously. About 2 weeks later, the tumour could be touched. We then monitored the tumour size every day, calculating the tumour volume using the formula $V=1 / 2 a \times b 2(a$ represents length, $b$ represents width). After the mice were killed, the tissue samples were fixed, processed as paraffin tissue sections, and used for immunohistochemistry and H\&E analysis.

Statistical analysis. Values were expressed as mean \pm s.d. The Student's $t$ and ANOVA tests were used to calculate $P$-values. $P$-values were two-sided, and considered statistically significant when $P<0.05$.

\section{RESULTS}

Sox 5 is induced by TGF- $\beta /$ Smad 3 signalling. Transforming growth factor-beta signalling has been associated with the invasion of cancer cells and metastasis, and it is a crucial regulator of EMT (Thakur et al, 2014; Buczek et al, 2016). We conjectured a relation between Sox 5 and TGF- $\beta$, so we treated LNCaP cells with TGF- $\beta$ and found that Sox5 expression increased gradually as well as $\mathrm{N}$-cadherin (Figure 1A). The mRNA of Sox 5 also increased with the addition of TGF- $\beta$ (Figure 1B). As a transcription factor, Sox 5 mainly resides in the nucleus and regulates gene expression by binding to 


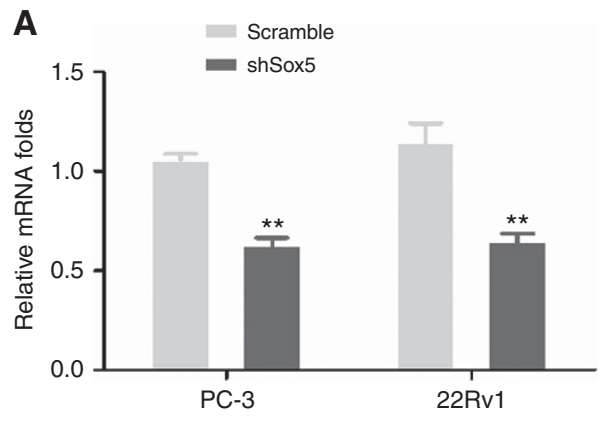

C

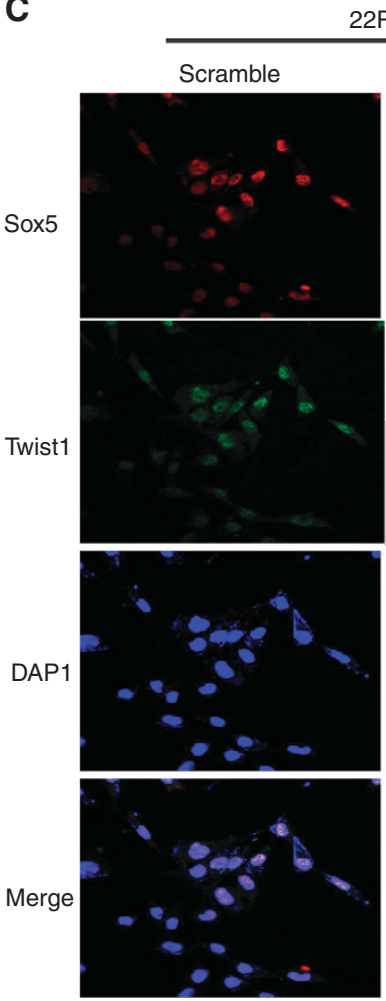

D

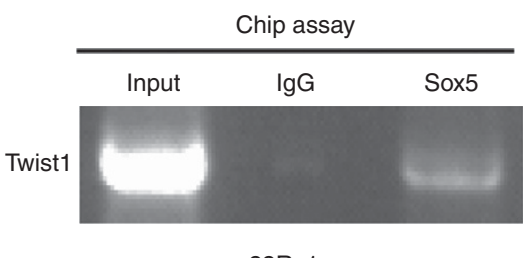

B
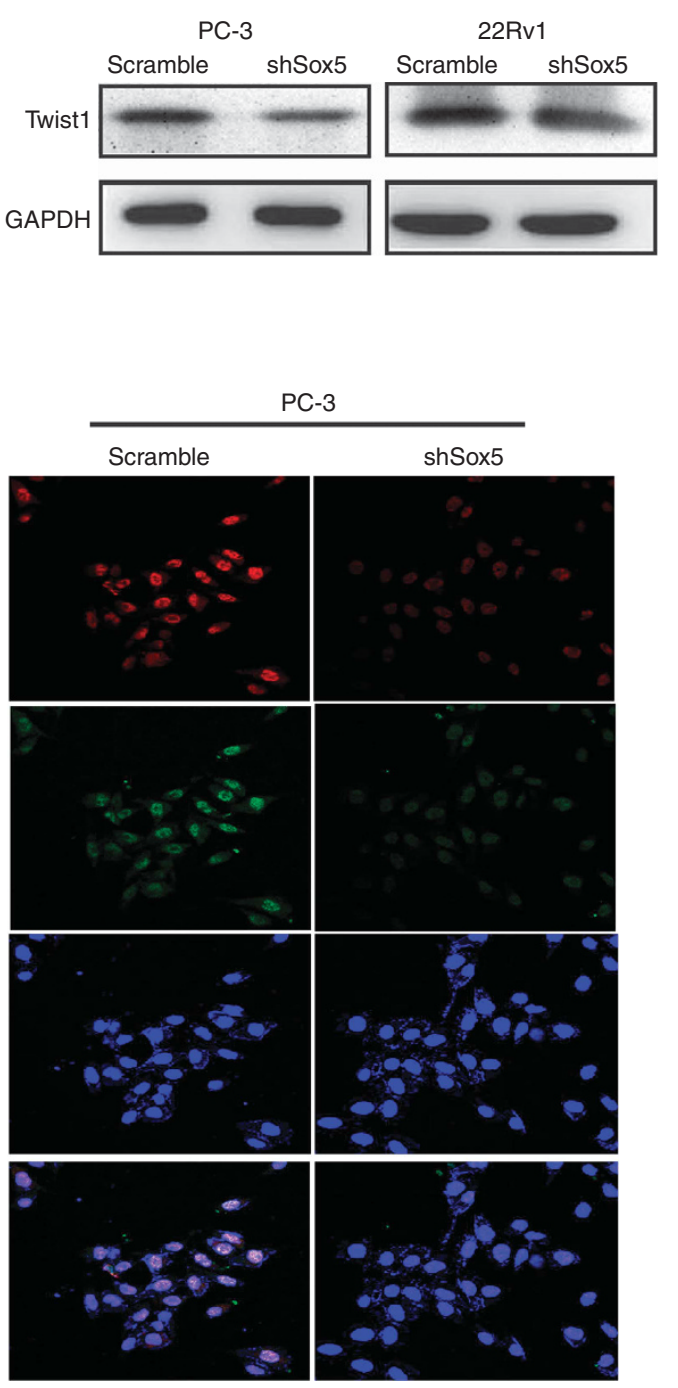

E

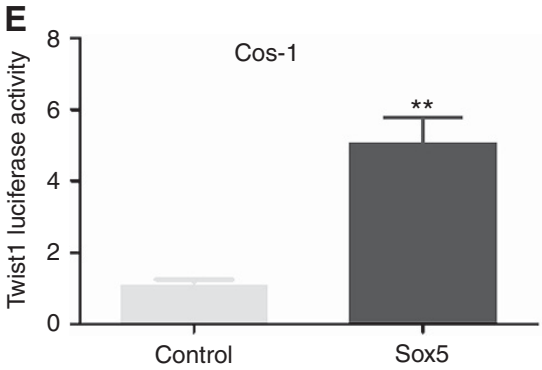

Figure 3. Sox 5 regulates EMT transcription factor Twist1 expression. (A) PC-3 and 22Rv1 cells were transfected with control or shSox5 plasmids, Sox 5 mRNA were detected by Q-PCR. (B) Twist1 expression in PC-3 and 22Rv1 cells transfected with control or shSox 5 plasmids by immunoblotting. (C) Twist1 and Sox5 expression in PC-3 and 22Rv1 cells transfected with control or shSox5 plasmids by immunofluorescence. (D) CHIP-PCR was performed in 22Rv1 cells with IgG as a negative control, showing that Sox 5 can bind to the Twist1 promoter. (E) Transfected Twist1 promoter plasmids with or without Sox5 plasmid in Cos-1 cells, then, luciferase values were detected. ${ }^{\star \star} P<0.01$.

enhancer or promoter regions (Mata-Rocha et al, 2014; Liu and Lefebvre, 2015). Nuclear protein extraction determined that Sox5 increased predominantly in the nucleus (Figure 1C). As TGF- $\beta$ can induce EMT through Sma- and Mad-related family 2 and 3 (SMAD2 and SMAD3) phosphorylation (Thakur et al, 2014; Buczek et al, 2016), we used JASPAR once again to determine the mechanisms of how TGF- $\beta$ induces Sox5 expression. We chose to test if Smad3 binds to the Sox 5 promoter. Surprisingly, positive results retrieved from JASPAR, as well as Smad3 CHIP in LNCaP after TGF- $\beta$ treatment for $24 \mathrm{~h}$, verified the prediction (Figure $1 \mathrm{D}$ ). To verify Smad3 functional binding to the Sox 5 promoter, we amplified a length of 459-bp Sox 5 promoter fragments in the CW22RV1 genome, cloned them into the pGL4.27 promoter luciferase vector, and transfected the constructed plasmid into LNCaP. Transforming growth factor-beta treatment increased luciferase intensities (Figure 1E). 
A
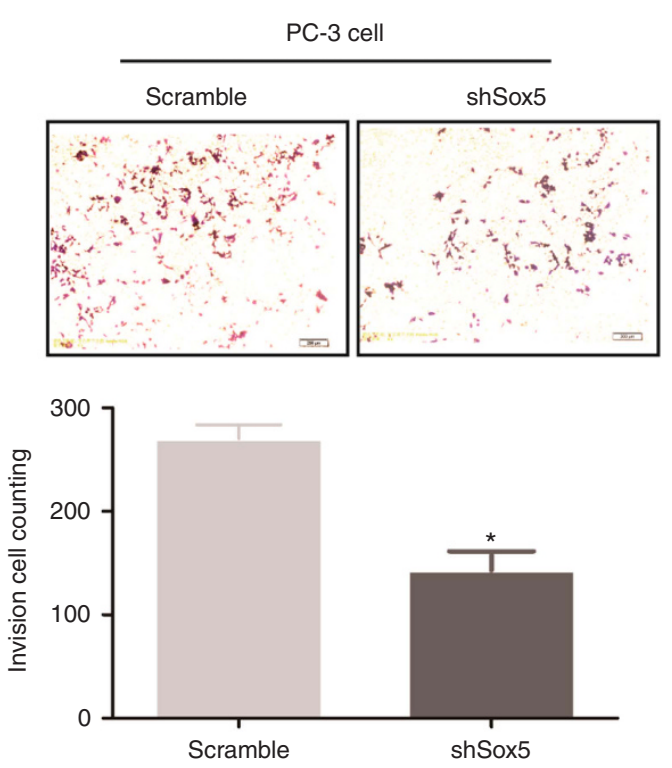

B
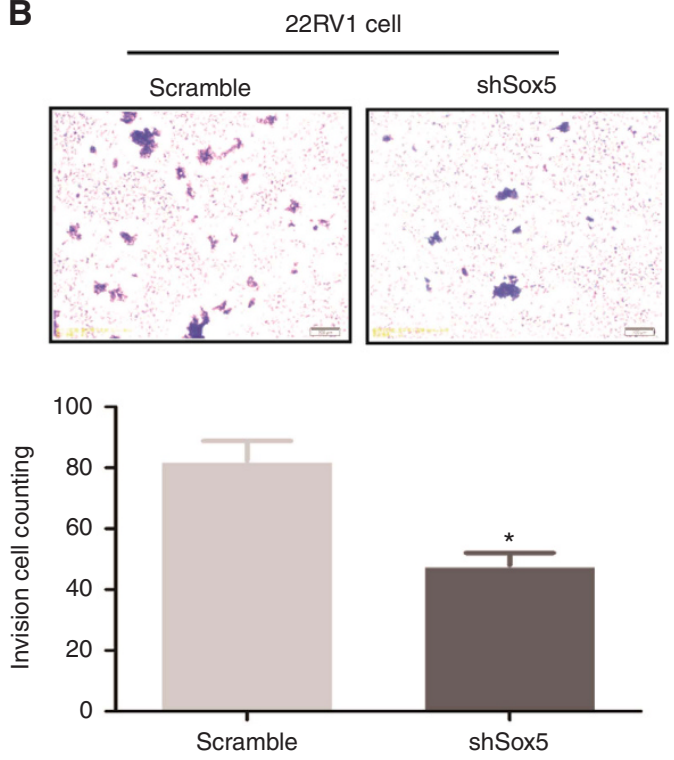

D
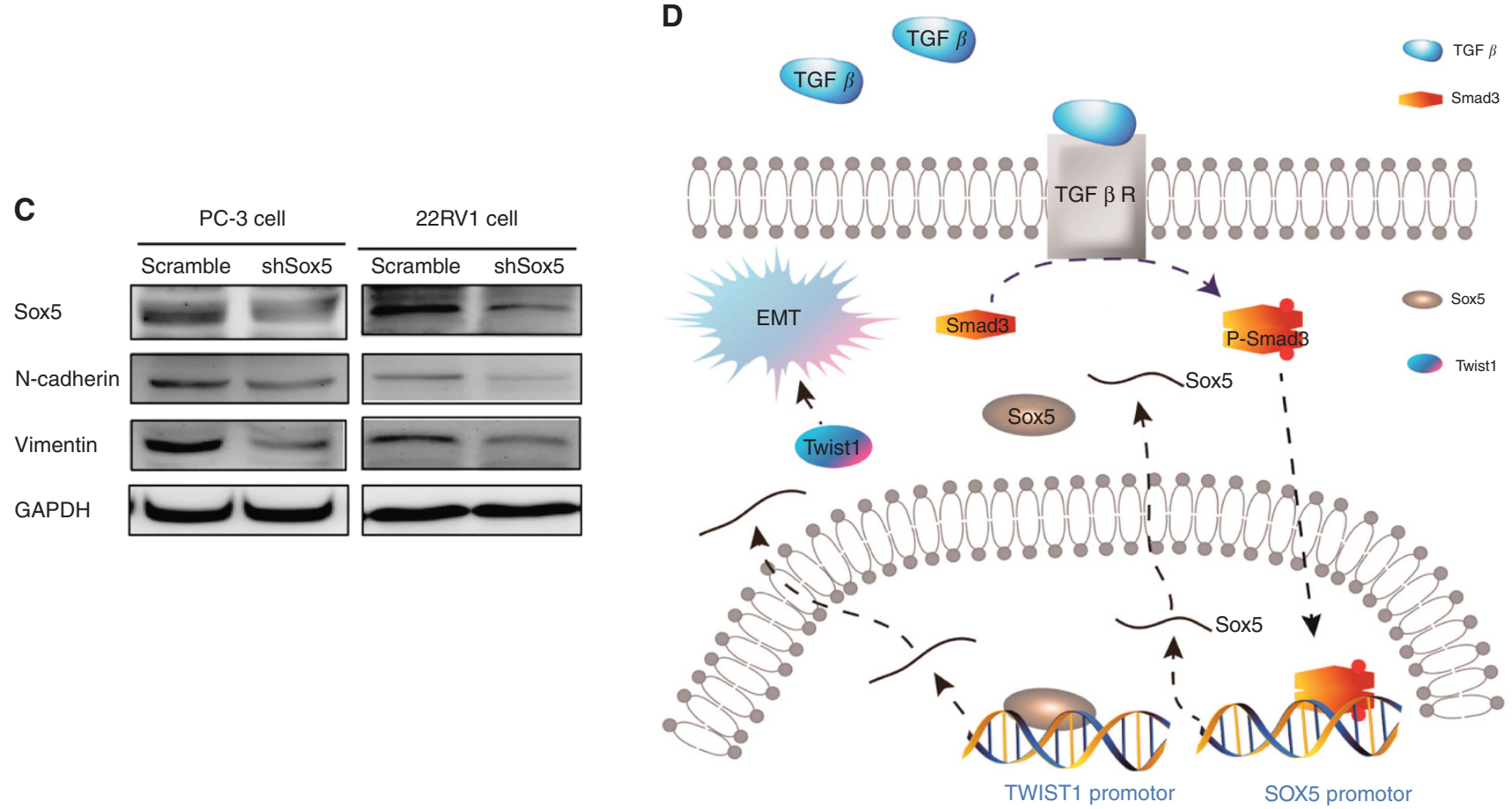

Figure 4. Targeting Sox5 will inhibit PCa cell migration and EMT in vitro. (A) Images of the invasion ability of control and shSox5 PC-3, and migrated cells were counted. (B) Images of the invasion ability of control and shSox5 22RV1, and migrated cells were counted. (C) Western blot shows that mesenchymal marker N-cadherin and vimentin expression was decreased in shSox5-PC-3 and shSox5-22RV1. (D) Schematic diagram of the Smad3-Sox5-Twist1 axis shows EMT regulating. ${ }^{*} P<0.05$.

Sox 5 is responsible for TGF- $\beta$-induced EMT and metastasis. To further explore the role of Sox 5 in TGF- $\beta$-induced EMT, we treated LNCaP with TGF- $\beta$ and knocked down Sox5 simultaneously. When we treat LNCaP cell with TGF- $\beta$, Sox 5 and $\mathrm{N}$-cadherin expressions were increased simultaneously. However, E-cadherin expression was decreased. Then we knocked down Sox5 after TGF- $\beta$ treatment, accompanied by $\mathrm{N}$-cadherin reduction and E-cadherin increase (Figure $2 \mathrm{~A}$ ). The above results were further to be identified by immunofluorescence assay (Figure 2B). EMT regulator, Twist1, was also detected by immunofluorescence assay. The result was the same as $\mathrm{N}$-cadherin that Sox 5 could also reverse TGF- $\beta$-induced Twistl expression (Figure 2C). We then performed a migration assay to observe cell mobility in various conditions. Transforming growth factor-beta increased LNCaP cell mobility, and knocking down Sox5 dampened the effect (Figure 2D). Cell counts indicated a significant difference between the shSox 5 and vehicle groups (Figure 2E).

Sox5 regulates EMT transcription factor Twist1 expression. In order to find the downstream gene of Sox 5 related to EMT regulation, we knockdown Sox 5 expression in CWR22RV1 and PC-3 cells, which have high expression of Sox 5 (Figure 3A). Previous data suggest that Sox 5 regulates Twist1 expression in hepatocellular carcinoma and breast cancer (Pei et al, 2014; Wang et al, 2015). We speculated that Sox 5 also regulates Twist 1 expression in PCa. When we knocked down Sox5, Twist1 expression also downregulated in CWR22RV1 and PC-3 cells (Figure 3B). Immunofluorescence suggested a reliable Sox5 knockdown effect and confirmed the downregulation of Twist1 (Figure 3C). A prediction from JASPAR 
A

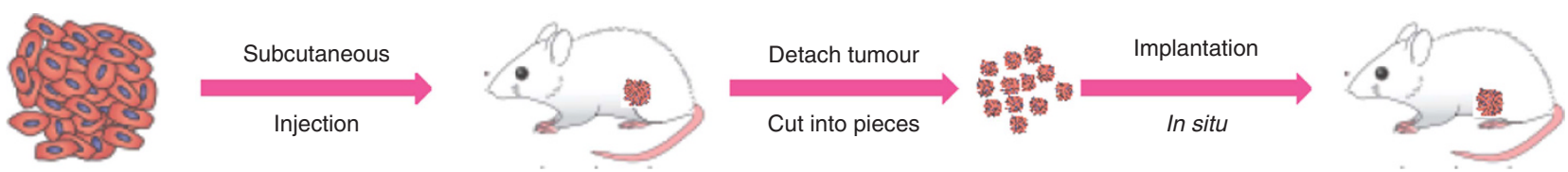

B

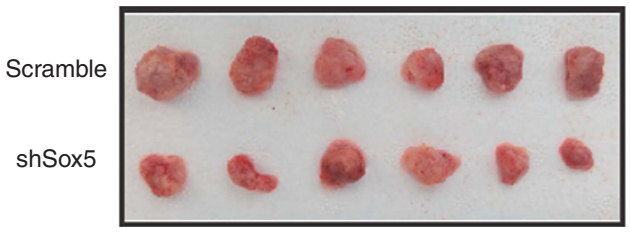

C
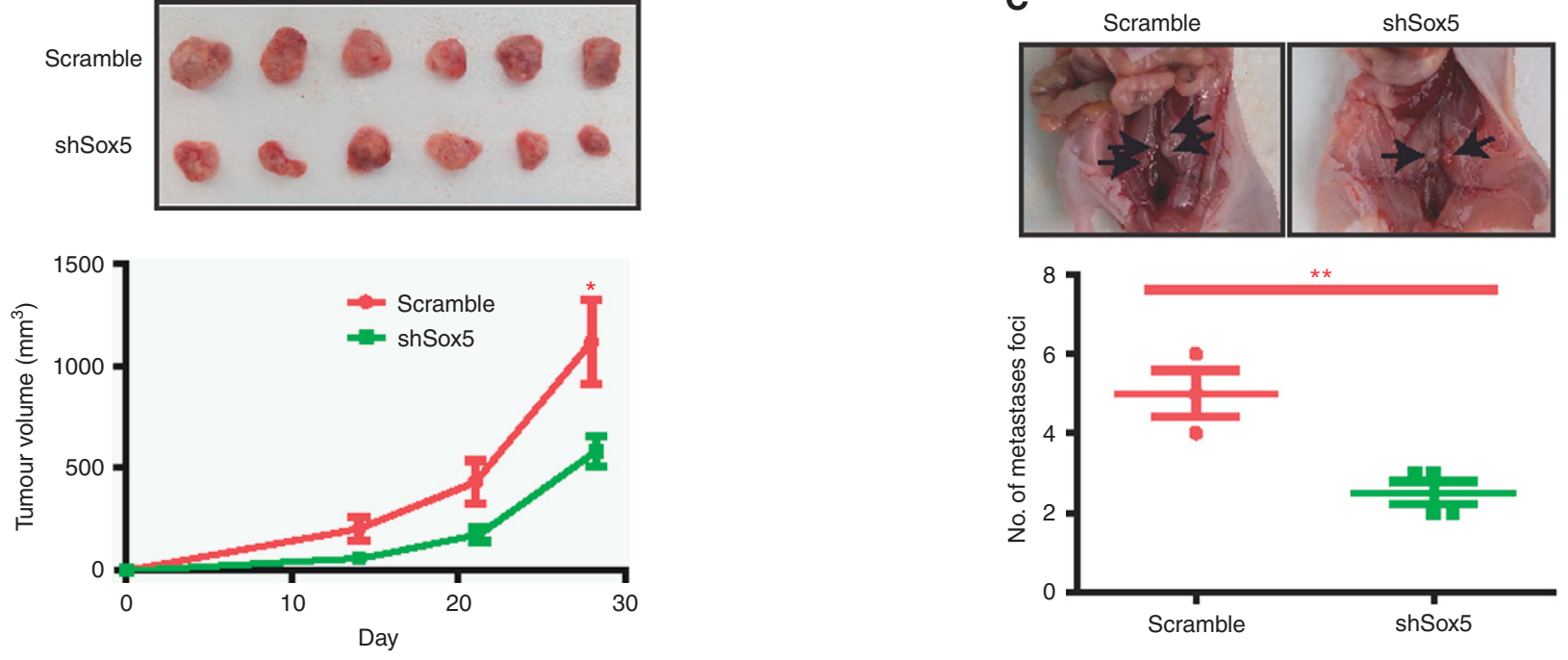

D

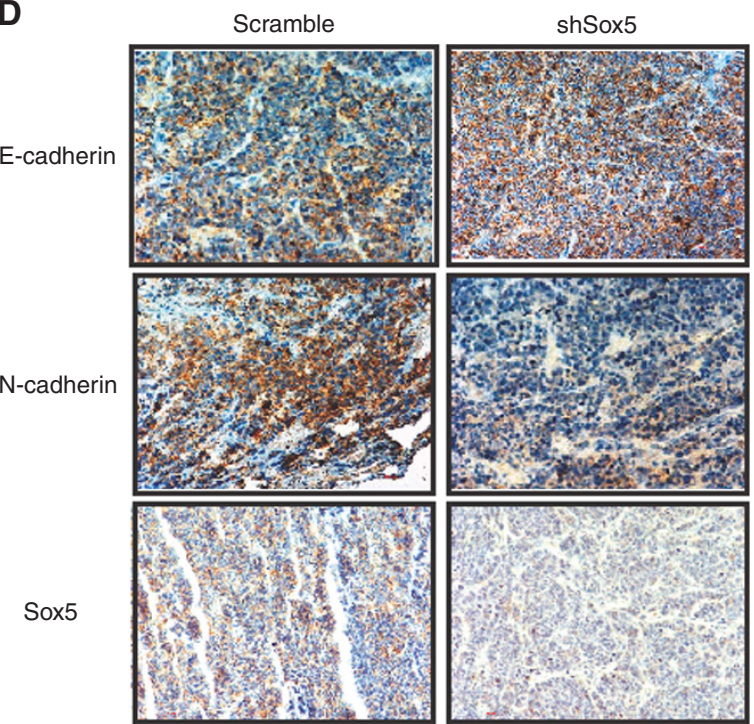

E

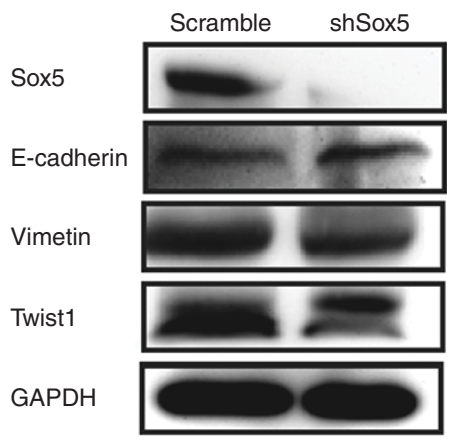

Figure 5. Target Sox5 inhibits prostate cancer progression in a xenograft mouse model. (A) Schematic diagram of the procedure. 22Rv1 cells were transfected with vehicle or shSox 5 plasmid and injected subcutaneously. Approximately 3 weeks later, the tumours were detached and cut into pieces, then were implanted orthotopically and subcutaneously. (B) Subcutaneous tumour growth was monitored (down). The mice were killed and the tumours were separated from mice (top). (C) Lymph node metastases are indicated (black arrows, top). The numbers of metastases were calculated (down). (D) IHC was applied to evaluate Sox5, E-cadherin, and N-cadherin expression. (E) WB was applied to evaluate Sox5, E-cadherin, Vimentin, and Twist1 expression. ${ }^{\star} P<0.05,{ }^{\star \star} P<0.01$.

(http://jaspar.genereg.net/) indicated that Sox5 can bind to the Twistl promoter region. We performed CHIP assay in CW22RV1 and confirmed that Sox 5 can bind to the Twist1 promoter (Figure 3D). To verify that the binding is functional, we amplified a length of 626-bp Twist1 promoter fragments in the CW22RV1 genome and cloned them into a pGL4.27 promoter luciferase vector. Co-transfection of the constructed plasmid and the Sox 5 overexpression plasmid into Cos-1 increased the luciferase intensities compared with the constructed plasmid and the vehicle plasmid (Figure 3E). Thus, we suggest that Sox 5 regulates EMT transcription factor Twist1 expression, which may be responsible for Sox5-induced EMT.
Hence, the Smad3-Sox5-Twist1 axis plays a vital role in EMT, and the direct action of Smad3-Sox5 and Sox5-Twist1 interaction indicate that Sox 5 is the mainstay of the axis.

The effects of Sox 5 on prostate cancer cell mobility. We then performed migration assays to test cell mobility. The migration assay indicated that PC-3 and CWR22RV1 cells have decreased mobility after inhibiting Sox5 expression, and the number of cells migrating through the transwell was much less than in the control (Figure 4A and $\mathrm{B}$ ). WB also suggested that $\mathrm{N}$-cadherin and vimentin expression reduced in these two prostate cancer cells with lower Sox5 expression (Figure 4C). 
A
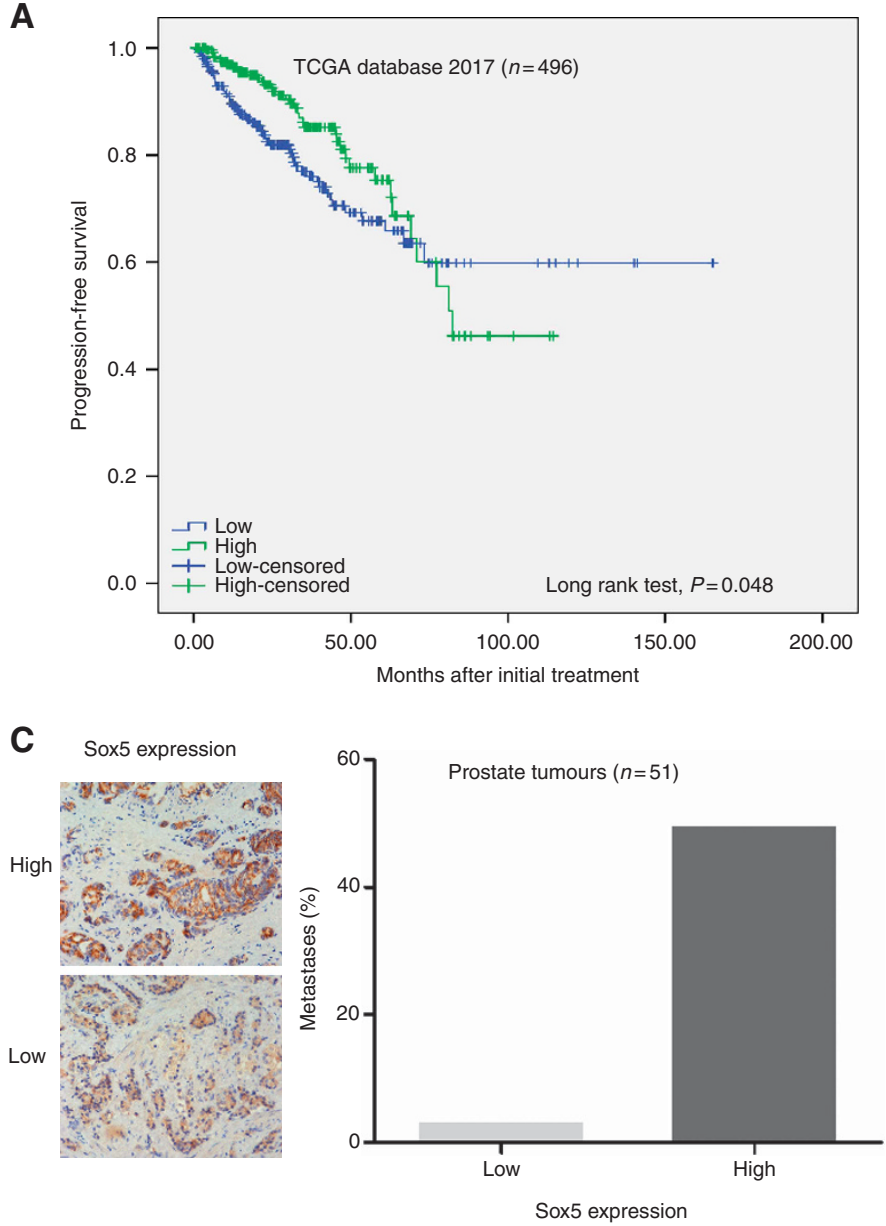

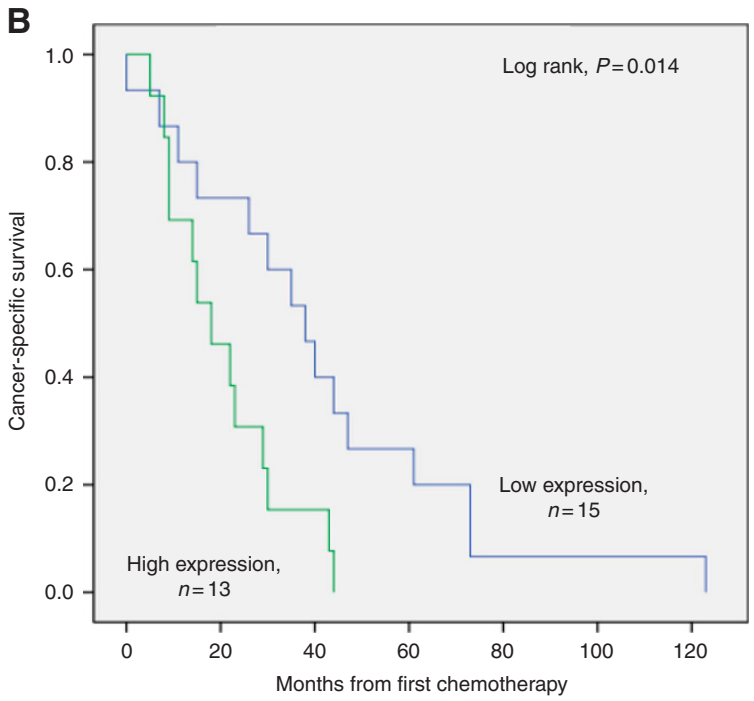

D

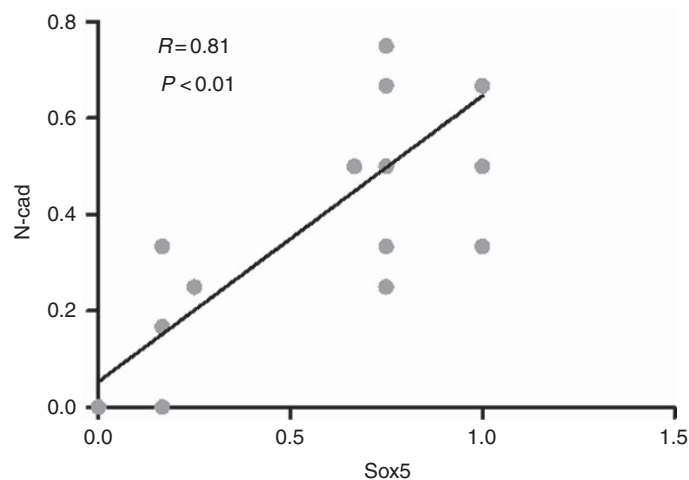

Figure 6. Sox5 is required for prostate cancer metastasis. (A) Progression-free survival of the TCGA prostate cancer patients with high or low levels of Sox 5 expression. (B) Cancer-specific survival of the metastatic prostate cancer patients with high or low levels of Sox 5 expression in Grasso et al. (2012). (C) Fifty-one samples were stratified into low and high Sox5 expression groups with a cut-off value of $0.5 \mathrm{intensity} \mathrm{(left).} \mathrm{The}$ metastases rates in low and high Sox 5 expression groups were compared by Chi-square test of the four table (right). (D) Sox 5 expression was positively correlated with $\mathrm{N}$-cadherin expression with a correlation coefficient of 0.81 .

The schematic diagram shows that the Smad3-Sox5-Twist1 axis invoices EMT regulating and contributes to PCa metastasis (Figure 4D).

Targeting Sox5 inhibits PCa progression in xenograft mouse model. In vitro data suggest that Sox 5 promotes PCa progression via EMT, so we completed in vivo experiments to corroborate this. We prepared approximately $1 \times 10^{7}$ vehicle and shSox 5 CW22RV1 cells to inject into subcutaneous tissues. Approximately 3 weeks later, the tumours were detached and cut into pieces and orthotopic and subcutaneous implantation were performed to observe tumour growth and metastases (Figure 5A). Subcutaneous tumours can be palpable approximately 2 weeks after implantation, and their growth was monitored every other day. Data showed that the tumours were much smaller in the Sox 5 knockdown group (Figure 5B). To evaluate metastases, we dissected the mice and found enlargement of the abdominal aortic lymph node (Figure 5C). After counting the number of metastatic foci, we found that knockdown of Sox 5 can repress tumour metastases (Figure 5C). IHC showed that tumours in the shSox 5 group expressed low levels of Sox 5 and N-cadherin, but high levels of E-cadherin (Figure 5D). WB in tumour samples identified that lower mesenchymal markers expression, such as Vimentin and Twist1, accompanied with higher epithelial marker expression, such as E-cadherin (Figure 5E).
Together, in vitro and in vivo data suggest that Sox 5 promotes EMT and contributes to PCa metastases. Mechanistically, TGF- $\beta$ in the microenvironment induces Sox 5 expression via Smad3 phosphorylation. Twist1 is regulated by Sox5, resulting in EMT and metastasis in prostate cancer.

Sox 5 is correlated with metastasis in clinical prostate tumours. The correlation of Sox 5 expression with clinical progression was analysed for the TCGA cohort, and it was found that Sox5 elevation was linked to accelerated progression in prostate cancer (Figure 6A). In 28 prostate cancer patients with metastasis, low level of Sox 5 had better cancer-specific survival (Figure 6B). To further investigate the clinical relevance of Sox 5 , we examined a cohort $(n=51)$ of prostate cancer clinical samples collected from the Second Hospital of Tianjin Medical University by IHC staining. The expression of Sox 5 was stratified into low and high groups according to stain intensity and extent (Figure 6C, top). Combined analysis of the expression and clinical data showed that patients with high Sox5 expression had a propensity to suffer from metastases (Figure 6C, bottom). We also found that Sox 5 expression was highly correlated with $\mathrm{N}$-cadherin expression $(R=0.81, P<0.01$; Figure 6D).

Together, the results from clinical data and in vitro experiments suggested Sox 5 contributes to prostate cancer metastases through EMT. 


\section{DISCUSSION}

Prior studies show that Sox 5 plays an important role in the progression of various cancers. In prostate cancer, proof from TCGA database showed Sox 5 was correlated to prostate progression (Figure 6A). To further unclear the role of Sox 5 in prostate cancer progression, we studied the relationship between the level of Sox 5 and metastasis. As a result, we found that the patients with high level Sox5 was more likely to suffer from metastasis, which was consistent with previous research (Ma et al, 2009). In another cohort of 28 prostate cancer patients with metastasis, low level of Sox 5 had a better cancer-specific survival (Figure 6B). According to the clinic data, we conclude that Sox 5 will accelerate the death of late stage PCa patients. However, the function of Sox5 in PCa progression has not previously been explored. In our research, we found that Sox 5 was highly correlated with $\mathrm{N}$-cadherin expression. Sox 5 and EMT were linked by transcription factor Twist1 in our data. We further demonstrated that Sox 5 could regulate Twist1 expression by CHIP and luciferase assays. Twist1 is known to promote tumour metastasis through inducing EMT (Eckert et al, 2011). It is well known that TGF- $\beta$ can induce Twist1 expression; one mechanism is by Smadmediated expression of HMGA2 (Thuault et al, 2006). In the process of induction of chondrogenic differentiation of stromal stem cells by medium containing TGF- $\beta 3$, dexamethasone, and bone morphogenetic protein (BMP)-6, Sox5 expression was increased five folds (Sekiya et al, 2002). Considering the common relationship between TGF- $\beta$-Twist1/EMT and Sox5-Twist1/EMT, we speculated that Sox 5 can be induced by TGF- $\beta$. Evidence from our data confirmed that TGF- $\beta$ could induce Sox 5 expression via Smad3 phosphorylation. Targeting Sox 5 could retard EMT induced by TGF- $\beta$ signal. Thus, we propose a novel mechanism for Smad3-Sox5-Twist1 that involves TGF- $\beta$-induced EMT. The origins of TGF- $\beta$ include carcinoma-associated fibroblasts, extracellular matrix, and tumour cells (Ao et al, 2007). TGF- $\beta$ can act on tumour cells in an autocrine, paracrine, and sometimes endocrine manner (Ten Dijke and Arthur, 2007). There are many challenges to develop TGF- $\beta$ inhibitors due to the tumour microenvironment and its manner of action, and it is difficult to balance proliferation and metastases as TGF- $\beta$ has dual effects on cancer cell proliferation (Neuzillet et al, 2015). Our research prompted targeting Sox5, not only to inhibit EMT and metastases but also to repress PCa cell proliferation. It is worth of further study in future.

Overall, our results demonstrate that Sox 5 is a critical component of TGF- $\beta$ signalling. The uncovering of this TGF- $\beta$ Sox5-Twist1 axis will extend our comprehension of TGF- $\beta$ network complexity and argue for Sox 5 as a new option to target TGF- $\beta$ signalling for cancer intervention.

\section{ACKNOWLEDGEMENTS}

This work was supported by National Natural Science Foundation of China (81302211, 81472682, and 81772756) and Natural Science Foundation of Tianjin (17JCZDJC35300, 15JCZDJC35400, and 15JCYBJC27200). Statement of ethical approval: The study design and procedures were approved by the ethical Committee of Tianjin Medical University (Approval number: KY2017K010).

\section{CONFLICT OF INTEREST}

The authors declare no conflict of interest.

\section{REFERENCES}

Ao MF, Franco OE, Park D, Raman D, Williams K, Hayward SW (2007) Cross-talk between paracrine-acting cytokine and chemokine pathways promotes malignancy in benign human prostatic. Cancer Res 67: 4244-4253.

Buczek ME, Miles AK, Green W, Johnson C, Boocock DJ, Pockley AG, Rees RC, Hulman G, van Schalkwyk G, Parkinson R, Hulman J, Powe DG, Regad T (2016) Cytoplasmic PML promotes TGF- $\beta$-associated epithelialmesenchymal transition and invasion in prostate cancer. Oncogene 35(26): 3465-3475.

Eckert MA, Lwin TM, Chang AT, Kim J, Danis E, Ohno-Machado L, Yang J (2011) Twist1-induced invadopodia formation promotes tumor metastasis. Cancer Cell 19: 372-386.

Grasso CS, Wu YM, Robinson DR, Cao X, Dhanasekaran SM, Khan AP, Quist MJ, Jing X, Lonigro RJ, Brenner JC, Asangani IA, Ateeq B, Chun SY, Siddiqui J, Sam L, Anstett M, Mehra R, Prensner JR, Palanisamy N, Ryslik GA, Vandin F, Raphael BJ, Kunju LP, Rhodes DR, Pienta KJ, Chinnaiyan AM, Tomlins SA (2012) The mutational landscape of lethal castrationresistant prostate cancer. Nature 487: 239-243.

Hodson R (2015) Small organ, big reach. Nature 528: S118-S119.

Huang DY, Lin YT, Jan PS, Hwang YC, Liang ST, Peng Y, Huang CY, Wu HC, Lin CT (2008) Transcription factor SOX-5 enhances nasopharyngeal carcinoma progression by down-regulating SPARC gene expression. J Pathol 214: 445-455.

Hussain M, Le Moulec S, Gimmi C, Bruns R, Straub J, Miller K, Group P.S (2016) Differential effect on bone lesions of targeting integrins: randomized phase II trial of abituzumab in patients with metastatic castration-resistant prostate cancer. Clin Cancer Res 22(13): 3192-3200.

Ikeda T, Kawaguchi H, Kamekura S, Ogata N, Mori Y, Nakamura K, Ikegawa S, Chung UI (2005) Distinct roles of Sox5, Sox6, and Sox9 in different stages of chondrogenic differentiation. J Bone Miner Metab 23: 337-340.

Jadaan DY, Jadaan MM, McCabe JP (2015) Cellular plasticity in prostate cancer bone metastasis. Prostate Cancer 2015: 651580.

Jiang WG, Sanders AJ, Katoh M, Ungefroren H, Gieseler F, Prince M, Thompson SK, Zollo M, Spano D, Dhawan P, Sliva D, Subbarayan PR, Sarkar M, Honoki K, Fujii H, Georgakilas AG, Amedei A, Niccolai E, Amin A, Ashraf SS, Ye L, Helferich WG, Yang X, Boosani CS, Guha G, Ciriolo MR, Aquilano K, Chen S, Azmi AS, Keith WN, Bilsland A, Bhakta D, Halicka D, Nowsheen S, Pantano F, Santini D (2015) Tissue invasion and metastasis: molecular, biological and clinical perspectives. Semin Cancer Biol 35(Suppl): S244-S275.

Kalluri R, Weinberg RA (2009) The basics of epithelial-mesenchymal transition. J Clin Invest 119: 1420-1428.

Lefebvre V, Behringer RR, de Crombrugghe B (2001) L-Sox5, Sox6 and Sox9 control essential steps of the chondrocyte differentiation pathway. Osteoarthritis Cartilage 9(Suppl A): S69-S75.

Lefebvre V, Dumitriu B, Penzo-Mendez A, Han Y, Pallavi B (2007) Control of cell fate and differentiation by Sry-related high-mobility-group box (Sox) transcription factors. Int J Biochem Cell Biol 39: 2195-2214.

Liu CF, Lefebvre V (2015) The transcription factors SOX9 and SOX5/SOX6 cooperate genome-wide through super-enhancers to drive chondrogenesis. Nucleic Acids Res 43: 8183-8203.

Ma S, Chan YP, Woolcock B, Hu L, Wong KY, Ling MT, Bainbridge T, Webber D, Chan TH, Guan XY, Lam W, Vielkind J, Chan KW (2009) DNA fingerprinting tags novel altered chromosomal regions and identifies the involvement of SOX5 in the progression of prostate cancer. Int $J$ Cancer 124: 2323-2332.

Massague J (2008) TGF- $\beta$ in cancer. Cell 134: 215-230.

Mata-Rocha M, Hernandez-Sanchez J, Guarneros G, de la Chesnaye E, Sanchez-Tusie AA, Trevino CL, Felix R, Oviedo N (2014) The transcription factors Sox 5 and Sox 9 regulate Catsper1 gene expression. FEBS Lett 588: 3352-3360.

Neuzillet C, Tijeras-Raballand A, Cohen R, Cros J, Faivre S, Raymond E, de Gramont A (2015) Targeting the TGF- $\beta$ pathway for cancer therapy. Pharmacol Therapeut 147: 22-31.

Pei XH, Lv XQ, Li HX (2014) Sox5 induces epithelial to mesenchymal transition by transactivation of Twist1. Biochem Biophys Res Commun 446: 322-327.

Pickup M, Novitskiy S, Moses HL (2013) The roles of TGF- $\beta$ in the tumour microenvironment. Nat Rev Cancer 13: 788-799. 
Renjie W, Haiqian L (2015) MiR-132, miR-15a and miR-16 synergistically inhibit pituitary tumor cell proliferation, invasion and migration by targeting Sox5. Cancer Lett 356: 568-578.

Sekiya I, Vuoristo JT, Larson BL, Prockop DJ (2002) In vitro cartilage formation by human adult stem cells from bone marrow stroma defines the sequence of cellular and molecular events during chondrogenesis. Proc Natl Acad Sci USA 99: 4397-4402.

Siegel RL, Miller KD, Jemal A (2017) Cancer statistics, 2017. CA Cancer J Clin 67(1): 7-30.

Steinberger AE, Ledet EM, Luk E, Cotogno P, Stolten M, Desmond D, Feibus A, Silberstein J, Sartor O (2016) Characterizations of clinical and therapeutic histories for men with prostate cancer-specific mortality. Clin Genitourin Cancer 14: 139-148.

Ten Dijke P, Arthur HM (2007) Extracellular control of TGF-beta signalling in vascular development and disease. Nat Rev Mol Cell Biol 8: 857-869.

Thakur N, Gudey SK, Marcusson A, Fu JY, Bergh A, Heldin CH, Landstrom M (2014) TGF- $\beta$-induced invasion of prostate cancer cells is promoted by c-Jundependent transcriptional activation of Snail. Cell Cycle 13: 2400-2414.

Thiery JP, Lim CT (2013) Tumor dissemination: an EMT affair. Cancer Cell 23: $272-273$.
Thuault S, Valcourt U, Petersen M, Manfioletti G, Heldin CH, Moustakas A (2006) Transforming growth factor-beta employs HMGA2 to elicit epithelial-mesenchymal transition. J Cell Biol 174: 175-183.

Tsai JH, Yang J (2013) Epithelial-mesenchymal plasticity in carcinoma metastasis. Genes Dev 27: 2192-2206.

Wang D, Han S, Wang X, Peng R, Li X (2015) SOX5 promotes epithelialmesenchymal transition and cell invasion via regulation of Twistl in hepatocellular carcinoma. Med Oncol 32: 461.

Wu KK (2006) Analysis of protein-DNA binding by streptavidin-agarose pulldown. Methods Mol Biol 338: 281-290.

Xie N, Cheng H, Lin D, Liu L, Yang O, Jia L, Fazli L, Gleave ME, Wang Y, Rennie P, Dong X (2015) The expression of glucocorticoid receptor is negatively regulated by active androgen receptor signaling in prostate tumors. Int J Cancer 136: E27-E38.

This work is published under the standard license to publish agreement. After 12 months the work will become freely available and the license terms will switch to a Creative Commons AttributionNonCommercial-Share Alike 4.0 Unported License. 